\title{
THRUST BEARING WITH ROUGH SURFACES LUBRICATED BY AN ELLIS FLUID
}

\author{
A. WALICKA*, E. WALICKI, P. JURCZAK and J. FALICKI \\ University of Zielona Góra, Faculty of Mechanical Engineering \\ ul. Szafrana 2, 65-516 Zielona Góra, POLAND \\ E-mails: A.Walicka@ijame.uz.zgora.pl \\ E.Walicki@ijame.uz.zgora.pl \\ P.Jurczak@ibem.uz.zgora.pl \\ J.Falicki@ibem.uz.zgora.pl
}

\begin{abstract}
In the paper the influence of bearing surfaces roughness on the pressure distribution and load-carrying capacity of a thrust bearing is discussed. The equations of motion of an Ellis pseudo-plastic fluid are used to derive the Reynolds equation. After general considerations on the flow in a bearing clearance and using the Christensen theory of hydrodynamic rough lubrication the modified Reynolds equation is obtained. The analytical solutions of this equation for the cases of a squeeze film bearing and an externally pressurized bearing are presented. As a result one obtains the formulae expressing pressure distribution and load-carrying capacity. A thrust radial bearing is considered as a numerical example.
\end{abstract}

Key words: pseudo-plastic fluid, Ellis model, thrust bearing, Christensen roughness.

\section{Introduction}

Steady state radial flows and time-dependent squeezing flows of viscoplastic fluids are encountered in a variety of fields (Covey and Stanmore, 1981; Dai and Bird, 1981; Lipscomb and Denn, 1984). These flows are found in fabrication operations such as stamping, injection moulding, and sheet forming. Material properties of highly viscous fluids are measured with a device called the 'plastometer' which incorporates a parallel-disk squeeze flow geometry (Covey and Stanmore, 1981). In addition, such flows are encountered in lubrication systems, and there is a considerable interest as to the degree in which viscoplastic additives enhance the load-bearing capacity of a lubricant. Finally, the study of these flows can provide a rigorous test of rheological constitutive equations since the flows involve a combination of elongation and shearing effects.

The flows of Newtonian fluids in the clearance of a thrust bearing with impermeable surfaces have been examined theoretically. The bearing walls have been modeled as two disks, two conical or spherical surfaces. A bearing formed by two surfaces of revolution constitutes a more general case of the problem (Walicka, 1989).

In recent years, a considerable amount of tribology research has been devoted to the study of the effect of surface roughness or geometric imperfections on hydrodynamic lubrication because the bearings surfaces, in practice, are all rough and the height of the roughness asperities may have the same order as the mean bearing clearance. Under these conditions, the surface roughness affects the bearing performance considerably.

The work in this area has mainly been confined to impermeable surfaces. The well-established stochastic theory of hydrodynamic lubrication of rough surfaces developed by Christensen (1970) formed the basis of this paper. In a series of works (Bujurke et al., 2007; Lin, 2000; 2001; Prakash and Tiwari, 1985;

\footnotetext{
* To whom correspondence should be addressed
} 
Walicka 2009; 2012; Walicka and Walicki, 2002a; 2002b) the model was applied to the study of the surface roughness of various geometrical configurations.

The modified Reynolds equation (Gurujan and Prakash, 1999) applicable to two types of directional roughness structure were used by Walicka and Walicki (2002a; 2002b) to find bearing parameters for the squeeze film between two curvilinear surface.

In this paper the Ellis fluid model is used to describe the pseudo-plastic behaviour of a lubricant. The modified Reynolds equation is derived and its solution for the curvilinear thrust bearing is presented. According to the Christensen stochastic model (1970), different forms of Reynolds equations are derived to take account of various types of surface roughness. Analytical solutions for the film pressure are presented for the longitudinal and circumferential roughness patterns.

\section{Derivation of the modified Reynolds}

It may be assumed that lubricating oils and some greases, with a viscosity index improver added, exhibit the same characteristics as pseudo-plastic fluids. Rotem and Shinnar (1961) proposed a method for expressing empirically the relation between the stress and the shear rate as

$$
\frac{d \gamma}{d t}=\frac{\tau}{\mu}\left(1+\sum_{i=-1}^{n} k_{i} \tau^{2 i}\right) .
$$

Similar relations were proposed by Whorlow (1992)

$$
\mu \frac{d \gamma}{d t}=\sum_{i=0}^{n} k_{i} \tau^{2 i+1}
$$

or

$$
\tau=\sum_{i=0}^{n} k_{i}\left(\frac{d \gamma}{d t}\right)^{2 i+1}
$$

known as power series models.

Retaining only the first and the last order terms the relations (2.1) or (2.2) reduce to

$$
\mu \frac{d \gamma}{d t}=\tau+k_{n} \tau^{n}
$$

the model known as the Ellis model (1927).

In what follows we will use the original notation given by Ellis in the form

$$
\frac{d \gamma}{d t}=\frac{\tau}{\mu_{N}}=\frac{\tau}{\mu}\left[1+\left|\frac{\tau}{\tau_{1 / 2}}\right|^{n-1}\right]
$$

where $\tau_{1 / 2}$ is a characteristic shear stress at which $\mu=2 \mu_{N}\left(\mu_{N}\right.$ - Newtonian viscosity) and $n$ is an identical parameter in the model.

The three-dimensional notation of Eq.(2.5) may be expressed as (Walicka, 2002a; 2002b) 


$$
\mu \boldsymbol{A}_{1}=\boldsymbol{\Lambda}\left\{1+\frac{1}{\tau_{l / 2}^{n-1}} \Lambda^{n-1}\right\} \quad \text { where } \quad \Lambda=\left[\frac{1}{2} \operatorname{tr}\left(\Lambda^{2}\right)\right]^{\frac{1}{2}}
$$

is the magnitude of the second-order shear stress tensor $\boldsymbol{\Lambda}$, but $\boldsymbol{A}_{l}$ is the first Rivlin-Ericksen kinematic tensor.

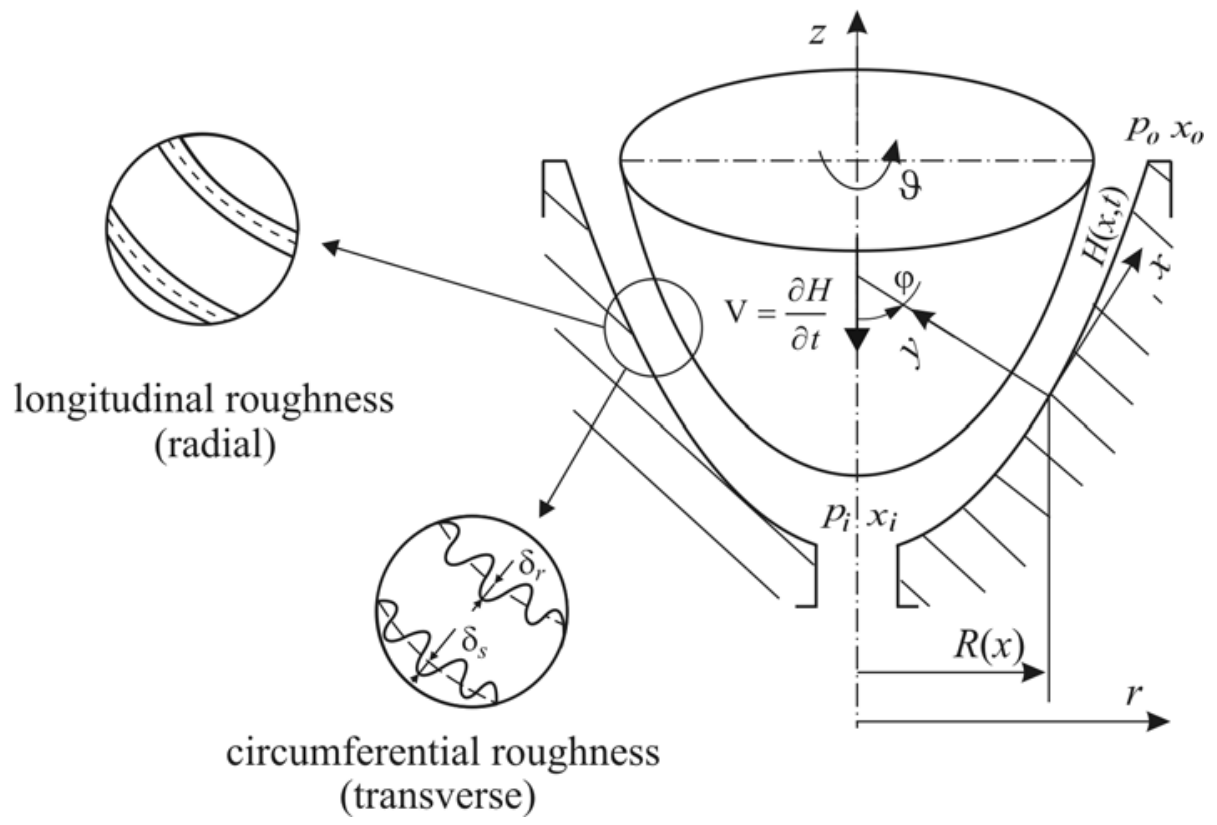

Fig.1. Geometry of a curvilinear thrust bearing.

Let us consider a thrust bearing with a curvilinear profile of the working surfaces shown in Fig.1. The lower surface is described by the function $R(x)$ which denotes the radius of this surface. The nominal bearing clearance thickness is given by the function $H(x, t)$.

The expression for the film thickness is considered to be made up of two parts.

$$
H=h(x, t)+h_{S}(x, \vartheta, \xi)
$$

where $h(x, t)$ represents the nominal smooth part of the film geometry, while $h_{s}=\delta_{r}+\delta_{s}$ denotes the random part resulting from the surface roughness asperities measured from the nominal level, $\xi$ describes a random variable which characterizes the definite roughness arrangement. An intrinsic curvilinear orthogonal coordinate system $x, \vartheta, y$ linked with the lower bearing surface is also presented in Fig.1.

Taking into account the considerations of the works (Walicka, 2002; Walicki, 2005) one may present the equation of continuity and the equations of motion of an Ellis fluid for axial symmetry in the form

$$
\begin{aligned}
& \frac{1}{R} \frac{\partial\left(R v_{x}\right)}{\partial x}+\frac{\partial v_{y}}{\partial y}=0, \\
& \frac{\partial \Lambda_{x y}}{\partial y}=\frac{\partial p}{\partial x}, \quad \frac{\partial p}{\partial y}=0 .
\end{aligned}
$$




$$
\mu \frac{\partial v_{x}}{\partial y}=\Lambda_{x y}\left[1+\frac{1}{\tau_{1 / 2}^{n-1}}\left|\Lambda_{x y}\right|^{n-1}\right]
$$

The problem statement is complete after specification of boundary conditions. These conditions for the velocity component are stated as follows:

$$
\begin{aligned}
& v_{x}(x, 0, t)=0, \quad v_{x}(x, H, t)=0, \\
& v_{y}(x, 0, t)=0, \quad v_{y}(x, h, t)=\frac{\partial H}{\partial t}=\dot{H},
\end{aligned}
$$

Solving the equations of motion (2.8), (2.9) and taking into account the constitutive equation (2.10) one obtains the modified Reynolds equation:

$$
\frac{1}{R} \frac{\partial}{\partial x} R H^{3}\left(-\frac{\partial p}{\partial x}\right)\left[1+\frac{3}{n+2}\left(\frac{H}{2 \tau_{1 / 2}}\right)^{n-1}\left(-\frac{\partial p}{\partial x}\right)^{n-1}\right]=-12 \mu \frac{\partial h}{\partial t}
$$

for a lubricating pseudo-plastic fluid of Ellis type. At $n=1-$ taking into consideration that $\mu=2 \mu_{N}-$ the above equation stands identical to the Reynolds equation for a Newtonian fluid (Walicki, 1977).

If the film thickness is regarded as a random quantity, a height distribution function must be associated. Many real bearing surfaces show a roughness height distribution which is closely Gaussian, at least up to three standard deviations. From a practical point of view, the Gaussian distribution is rather inconvenient and therefore a polynomial form of its approximation is chosen. Following Christensen (1970; $1971 ; 1973)$ such a probability density function is

$$
f\left(h_{s}\right)= \begin{cases}\frac{35}{32 c^{7}}\left(c^{2}-h_{s}^{2}\right)^{3}, & -c \leq h_{s} \leq+c \\ 0, & \text { elsewhere }\end{cases}
$$

where $c$ is the half total range of the random film thickness variable. The function terminates at $c= \pm 3 \sigma$, where $\sigma$ is the standard deviation.

Inserting expected values in Eq.(2.13) we get the general form of the stochastic Reynolds equation

$$
\frac{1}{R} \frac{\partial}{\partial x}\left(E\left\{R H^{3}\left(-\frac{\partial p}{\partial x}\right)\left[1+\frac{3}{n+2}\left(\frac{H}{2 \tau_{1 / 2}}\right)^{n-1}\left(-\frac{\partial p}{\partial x}\right)^{n-1}\right]\right\}\right)=-12 \mu \frac{\partial E(H)}{\partial t}
$$

or

$$
\frac{1}{R} \frac{\partial}{\partial x} R\left(E\left(H^{3}\right)\left[-\frac{\partial(E p)}{\partial x}\right]\left\{1+\frac{3 E\left(H^{n-1}\right)}{(n+2)\left(2 \tau_{1 / 2}\right)^{n-1}}\left[-\frac{\partial(E p)}{\partial x}\right]^{n-1}\right\}\right)=-12 \mu \frac{\partial E(H)}{\partial t}
$$


where $E(\cdot)$ is the expectancy operator defined by

$$
E(\cdot)=\int_{-c}^{+c}(\cdot) f\left(h_{s}\right) d h_{s}
$$

The values of the exponent $n$ for the Ellis fluid is included in the interval (Whorlow, 1992)

$$
0.25 \leq n \leq 4.0
$$

The problem is now reduced to devising means of evaluating the left-hand side of Eq.(2.15) subject to the specific model of roughness. The calculation of the mean film pressure distribution would require the evaluation of the expected value of various film thickness functions; these functions are given in the Appendix.

\section{Solutions to the modified Reynolds equation}

Consider the case of an Ellis fluid for which there is

$$
\frac{3}{n+2}\left(\frac{H}{2 \tau_{1 / 2}}\right)^{n-1}\left(-\frac{\partial \bar{p}}{\partial x}\right)^{n-1} \ll 1
$$

this relation allows us to search the solutions of the Reynolds equation (2.15) in the form of a sum

$$
E p=E p^{(0)}+E p^{(1)}
$$

which arrives at two linearized equations

$$
\frac{1}{R} \frac{\partial}{\partial x} R\left\{E\left(H^{3}\right)\left[-\frac{\partial\left(E p^{(0)}\right)}{\partial x}\right]\right\}=-12 \mu \frac{\partial E(H)}{\partial t},
$$

and

$$
\frac{1}{R} \frac{\partial}{\partial x} R\left\{E\left(H^{3}\right)\left[-\frac{\partial\left(E p^{(1)}\right)}{\partial x}\right]\right\}=-\frac{3}{n+2} \frac{1}{R} \frac{\partial}{\partial x} R\left\{\frac{E\left(H^{n+2}\right)}{\left(2 \tau_{1 / 2}\right)^{n-1}}\left[-\frac{\partial\left(E p^{(0)}\right)}{\partial x}\right]\right\}
$$

In this study two types of roughness will be of interest: the longitudinal (radial) one-dimensional roughness pattern, having the form of long narrow ridges and valleys running in the $x$ direction, and the circumferential (transverse) one-dimensional roughness pattern, having the form of long narrow ridges and valleys running in the $\vartheta$ direction (Walicka and Walicki, 2002a; 2002b; Walicka, 2009).

For the longitudinal one-dimensional roughness 


$$
H=h(x, t)+h_{s}(\vartheta, \xi)
$$

the stochastic Reynolds equation (3.3) and (3.4) remain without change, but for the circumferential onedimensional roughness

$$
H=h(x, t)+h_{s}(x, \xi)
$$

the stochastic Reynolds equations (3.3) and (3.5) take the form

$$
\frac{1}{R} \frac{\partial}{\partial x} R\left\{\frac{1}{E\left(H^{-3}\right)}\left[-\frac{\partial\left(E p^{(0)}\right)}{\partial x}\right]\right\}=-12 \mu \frac{\partial E(H)}{\partial t},
$$

and

$$
\begin{aligned}
& \frac{1}{R} \frac{\partial}{\partial x} R\left\{\frac{1}{E\left(H^{-3}\right)}\left[-\frac{\partial\left(E p^{(1)}\right)}{\partial x}\right]\right\}= \\
& =-\frac{3}{(n+2)\left(2 \tau_{1 / 2}\right)^{n-1}} \frac{1}{R} \frac{\partial}{\partial x} R\left\{\frac{1}{E\left(H^{-n-2}\right)}\left[-\frac{\partial\left(E p^{(0)}\right)}{\partial x}\right]^{n}\right\}
\end{aligned}
$$

Introducing the following relations

$$
H_{j}^{(3)}=\left\{\begin{array}{lll}
E\left(H^{3}\right) & \text { for } & j=l, \\
\frac{1}{E\left(H^{-3}\right)} & \text { for } \quad j=c,
\end{array} \quad H_{j}^{(n+2)}=\left\{\begin{array}{lll}
E\left(H^{(n+2)}\right) & \text { for } & j=l, \\
\frac{1}{E\left(H^{-n-2}\right)} & \text { for } \quad j=c
\end{array}\right.\right.
$$

we may present Eqs (3.3), (3.4) and Eqs (3.7), (3.8) in one common form as follows

$$
\begin{aligned}
& \frac{1}{R} \frac{\partial}{\partial x}\left[R H_{j}^{(3)}\left(-\frac{\partial \bar{p}^{(0)}}{\partial x}\right)\right]=-12 \mu \frac{\partial h}{\partial t}, \\
& \frac{1}{R} \frac{\partial}{\partial x}\left[R H_{j}^{(3)}\left(-\frac{\partial \bar{p}^{(1)}}{\partial x}\right)\right]=-\frac{3}{(n+2)\left(2 \tau_{1 / 2}\right)^{n-1}} \frac{1}{R} \frac{\partial}{\partial x}\left[R H_{j}^{(n+2)}\left(-\frac{\partial \bar{p}^{(0)}}{\partial x}\right)^{n}\right],
\end{aligned}
$$

where

$$
\bar{p}^{(0)}=E p^{(0)}, \quad \bar{p}^{(l)}=E p^{(l)}, \quad E(H)=h
$$


and the lower index $j=l$ refers to the one-dimensional longitudinal roughness, but $j=c$ refers to the onedimensional circumferential roughness.

The boundary conditions for pressure are:

- for a squeeze film bearing $\left(\frac{\partial h}{\partial t} \neq 0\right)$

$$
\left.\frac{\partial \bar{p}^{(0)}}{\partial x}\right|_{x=0}=0, \quad \bar{p}^{(0)}\left(x_{o}\right)=p_{o},\left.\quad \frac{\partial \bar{p}^{(l)}}{\partial x}\right|_{x=0}=\bar{p}^{(l)}\left(x_{o}\right)=0
$$

- for a externally pressurized bearing $\left(\frac{\partial h}{\partial t}=0\right)$

$$
\bar{p}^{(0)}\left(x_{i}\right)=p_{i}, \quad \bar{p}^{(0)}\left(x_{o}\right)=p_{o}, \quad \bar{p}^{(1)}\left(x_{i}\right)=\bar{p}^{(1)}\left(x_{o}\right)=0 .
$$

The solutions of Eqs (3.10) and (3.11) are given, respectively, as follows

$$
\bar{p}(x, t)=p_{o}-\left[F_{o}^{(n)}-F^{(n)}(x, t)\right]
$$

and

$$
\bar{p}=\alpha^{(n)} G^{(n)}(x)+\frac{\left[A(x)-A_{o}\right]\left(p_{i}-\alpha^{(n)} G_{i}^{(n)}\right)-\left[A(x)-A_{i}\right]\left(p_{o}-\alpha^{(n)} G_{o}^{(n)}\right)}{A_{i}-A_{o}}
$$

where

$$
\begin{aligned}
& I(x, t)=12 \mu \int \frac{\int R \dot{h} d x}{R H_{j}^{(3)}} d x, \\
& J^{(n)}(x, t)=\frac{3(12 \mu)^{n}}{(n+2)\left(2 \tau_{1 / 2}\right)^{n-1}} \int \frac{H_{j}^{(n+2)}}{R^{n}\left(H_{j}^{(3)}\right)^{n+1}}\left(-\int R \dot{h} d x\right)^{n} d x, \\
& F^{(n)}(x, t)=I(x, t)+J^{(n)}(x, t), \quad F_{o}=F\left(x_{o}, t\right) ; \\
& A(x)=\int \frac{d x}{R H_{j}^{(3)}}, \quad A_{i}=A\left(x_{i}\right), \quad \\
& C=\frac{p_{i}-p_{o}}{A_{i}-A_{o}}, \quad \alpha^{(n)}=\frac{3}{n+2} \frac{(-C)^{n}}{\left(2 \tau_{1 / 2}\right)^{n-1}}, \\
& G^{(n)}(x)=\int \frac{H_{j}^{(n+2)} d x}{R^{n}\left(H_{j}^{(3)}\right)^{n+1}}, \quad G_{i}^{(n)}=G^{(n)}\left(x_{i}\right), \quad G_{o}^{(n)}=G^{(n)}\left(x_{o}\right) .
\end{aligned}
$$


The load carrying capacity is defined, respectively, by

$$
N=2 \pi \int_{0}^{x_{o}}\left(\bar{p}-p_{o}\right) R \cos \varphi d x
$$

or

$$
N=\pi R_{i}^{2} p_{i}+2 \pi \int_{x_{i}}^{x_{o}} \bar{p} R \cos \varphi d x
$$

where the sense of angle $\varphi$ arises from Fig.1.

\section{Radial thrust squeeze film bearing}

Let us consider the radial thrust squeeze film bearing presented in Fig.2.

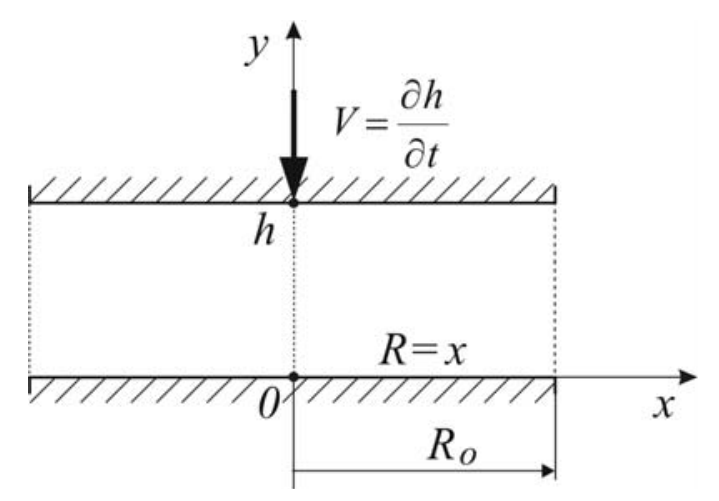

Fig.2. Radial squeeze film bearing.

Introducing the following parameters

$$
\begin{aligned}
& \tilde{x}=\frac{x}{R_{o}}, \quad x=R, \quad \tilde{R}=\frac{R}{R_{o}}, \quad \tilde{h}=\frac{h}{h_{o}}=e(t), \quad e(t)=1-\varepsilon(t), \quad c^{*}=\frac{c}{h_{o}}, \\
& \tilde{p}=\frac{\left(\bar{p}-p_{o}\right)}{\mu \dot{\varepsilon}}\left(\frac{h_{o}}{x_{o}}\right)^{2}, \quad \dot{\varepsilon}=\frac{d \varepsilon}{d t}, \quad \tilde{N}=\frac{N h_{o}^{2}}{\mu \dot{\varepsilon} x_{o}^{4}}, \quad \lambda^{(n)}=\left(\frac{\mu \dot{\varepsilon} x_{o}}{\tau_{1 / 2} h_{o}}\right)^{n-1} ;
\end{aligned}
$$

we have

$$
\begin{aligned}
& \tilde{p}=\frac{3}{\tilde{H}_{j}^{(3)}}\left[1-\tilde{x}^{2}-\frac{2 \cdot 3^{n} \lambda^{(n)}}{(n+1)(n+2)} \frac{\tilde{H}_{j}^{(n+2)}}{\left(\tilde{H}_{j}^{(3)}\right)^{n}}\left(1-\tilde{x}^{n+1}\right)\right], \\
& \tilde{N}=\frac{3 \pi}{2 \tilde{H}_{j}^{(3)}}\left[1-\frac{4 \cdot 3^{n} \lambda^{(n)}}{(n+2)(n+3)} \frac{\tilde{H}_{j}^{(n+2)}}{\left(\tilde{H}_{j}^{(3)}\right)^{n}}\right],
\end{aligned}
$$


To illustrate graphically these expressions let us take the values $n=2$ and $n=4$; introducing the notations

$$
\begin{aligned}
& \tilde{H}_{j}^{(3)}=\left\{\begin{array}{lll}
e^{3}\left[1+\frac{1}{3}\left(\frac{c^{*}}{e}\right)^{2}\right] & \text { for } & j=l \\
\left(\frac{1}{e^{3}}\left[1+\frac{2}{3}\left(\frac{c^{*}}{e}\right)^{2}\right]\right)^{-1} & \text { for } & j=c
\end{array}\right. \\
& \tilde{H}_{j}^{(4)}=\left\{\begin{array}{lll}
e^{4}\left[1+\frac{2}{3}\left(\frac{c^{*}}{e}\right)^{2}\right] \\
\left(\frac{1}{e^{4}}\left[1+\frac{10}{9}\left(\frac{c^{*}}{e}\right)^{2}\right]\right)^{-1} & \text { for } & j=l
\end{array}\right. \\
& \tilde{H}_{j}^{(6)}=\left\{\begin{array}{lll}
e^{6}\left[1+\frac{5}{93}\left(\frac{c^{*}}{e}\right)^{2}\right] & \text { for } & j=l \\
\left(\frac{1}{e^{6}}\left[1+\frac{7}{3}\left(\frac{c^{*}}{e}\right)^{2}\right]\right)^{-1} & \text { for } & j=c
\end{array}\right.
\end{aligned}
$$

we will present in Figs 3-6 the graphs of the pressure distributions and the load-carrying capacities.
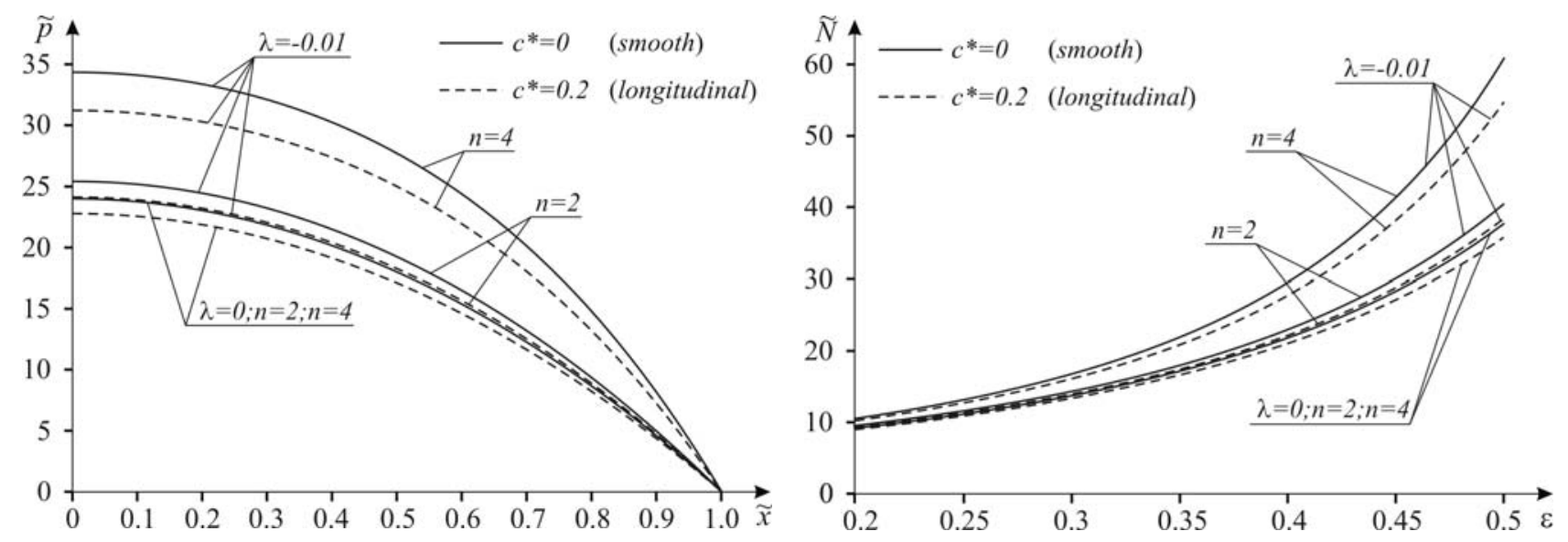

Fig.3. Dimensionless mechanical parameters of the thrust bearing with rough surfaces for longitudinal roughness for $\lambda=-0.01$ and $\lambda=0$ and $\varepsilon=0.5$; (a) pressure distribution and (b) load-carrying capacity. 

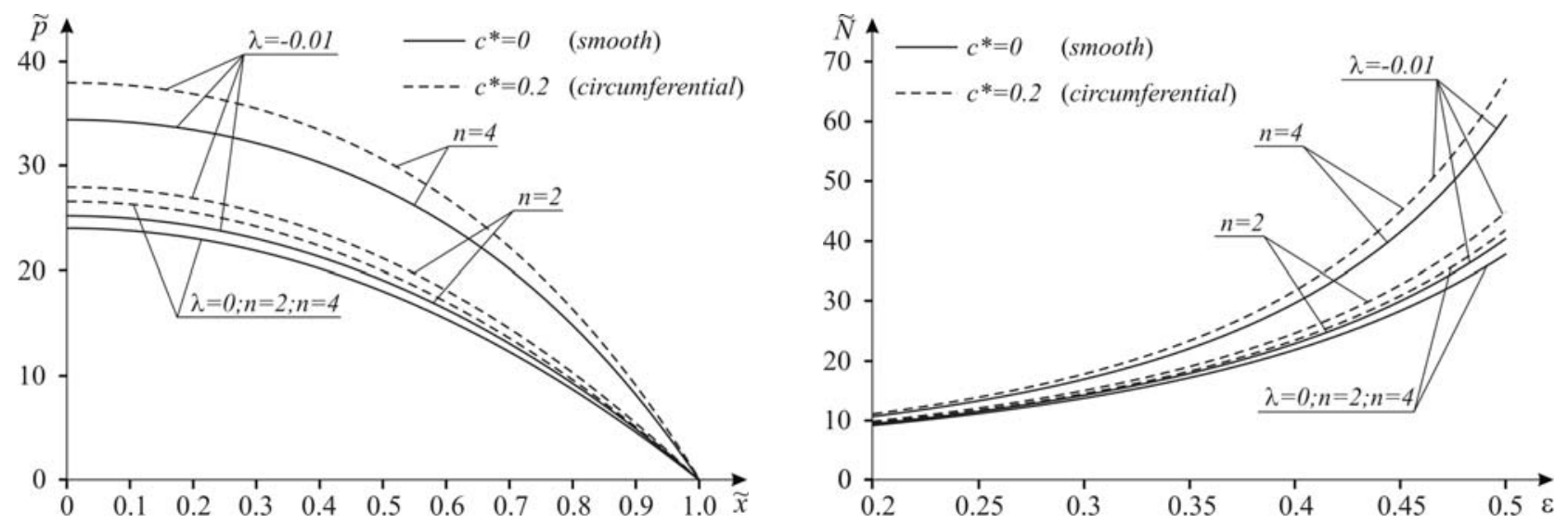

Fig.4. Dimensionless mechanical parameters of the thrust bearing with rough surfaces for circumferential roughness for $\lambda=-0.01$ and $\lambda=0$ and $\varepsilon=0.5$; (a) pressure distribution and (b) load-carrying capacity.
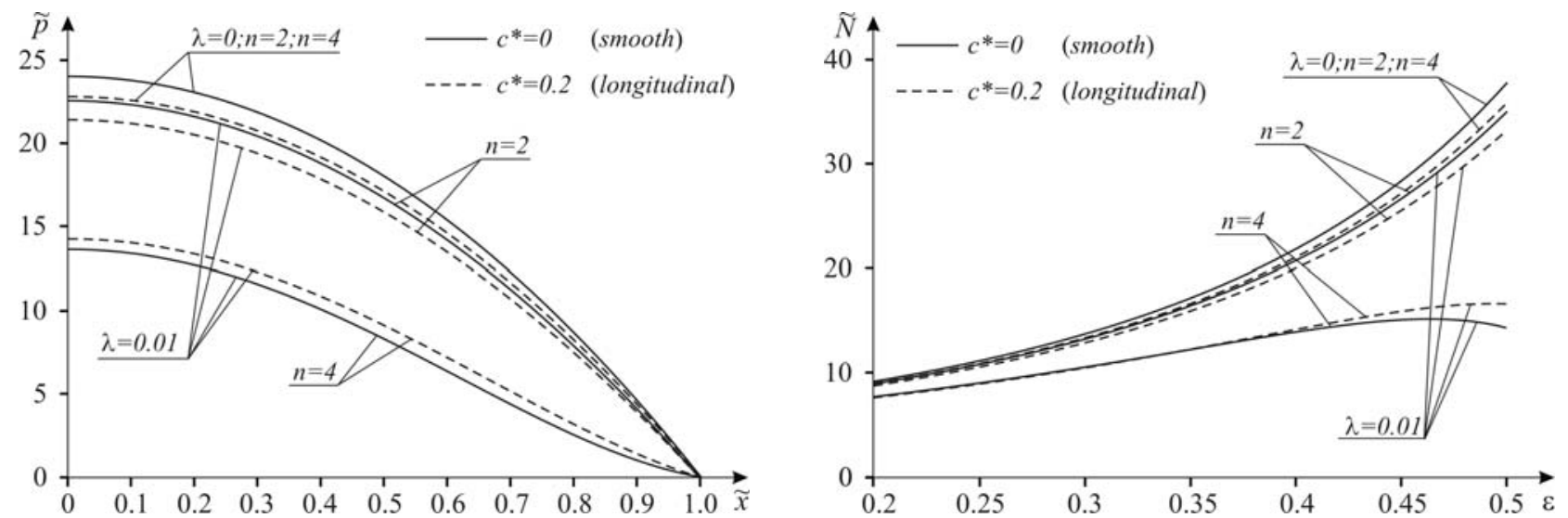

Fig.5. Dimensionless mechanical parameters of the thrust bearing with rough surfaces for longitudinal roughness for $\lambda=0$ and $\lambda=0.01$ and $\varepsilon=0.5$; (a) pressure distribution and (b) load-carrying capacity.
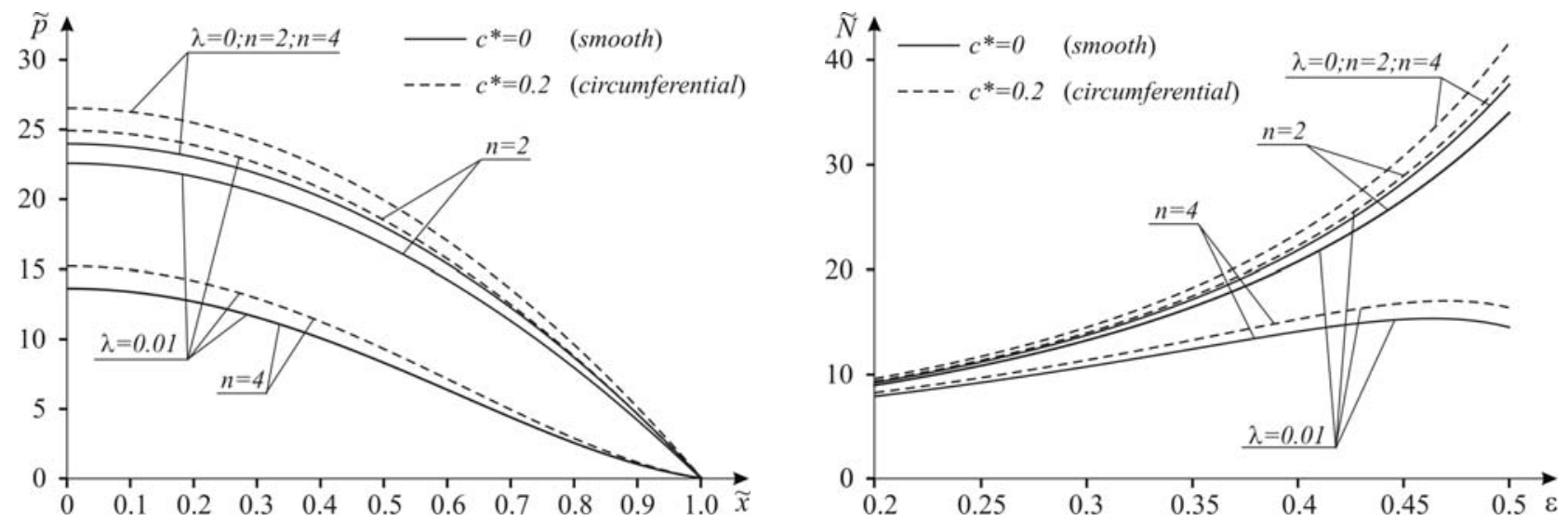

Fig.6. Dimensionless mechanical parameters of the thrust bearing with rough surfaces for circumferential roughness for $\lambda=0$ and $\lambda=0.01$ and $\varepsilon=0.5$; (a) pressure distribution and (b) load-carrying capacity. 


\section{Conclusions}

The modified Reynolds equation for an Ellis type of pseudo-plastic lubricants flowing in a clearance of a thrust curvilinear bearing with rough surfaces is derived. As a result the general formulae for pressure distributions and the load-carrying capacity are obtained. It follows from the calculations and their graphic presentations that both the magnitudes depend on the rheological parameters $\tau_{1 / 2}$ and $n$ or $\lambda$ and $n$. For a squeeze film radial bearing the pressures and load-carrying capacities increase with a decrease of the $\lambda$ values with respect to the values of Newtonian lubricants $(\lambda=0)$. These bearing parameters increase with an increase of $n$ for negative values of $\lambda$ and decrease for positive values of $\lambda$. The bearing surface roughness expressed by $c^{*}$, results in some small increase of the values of the bearing parameters for circumferential roughness and in a small decrease for longitudinal roughness.

\section{Nomenclature}

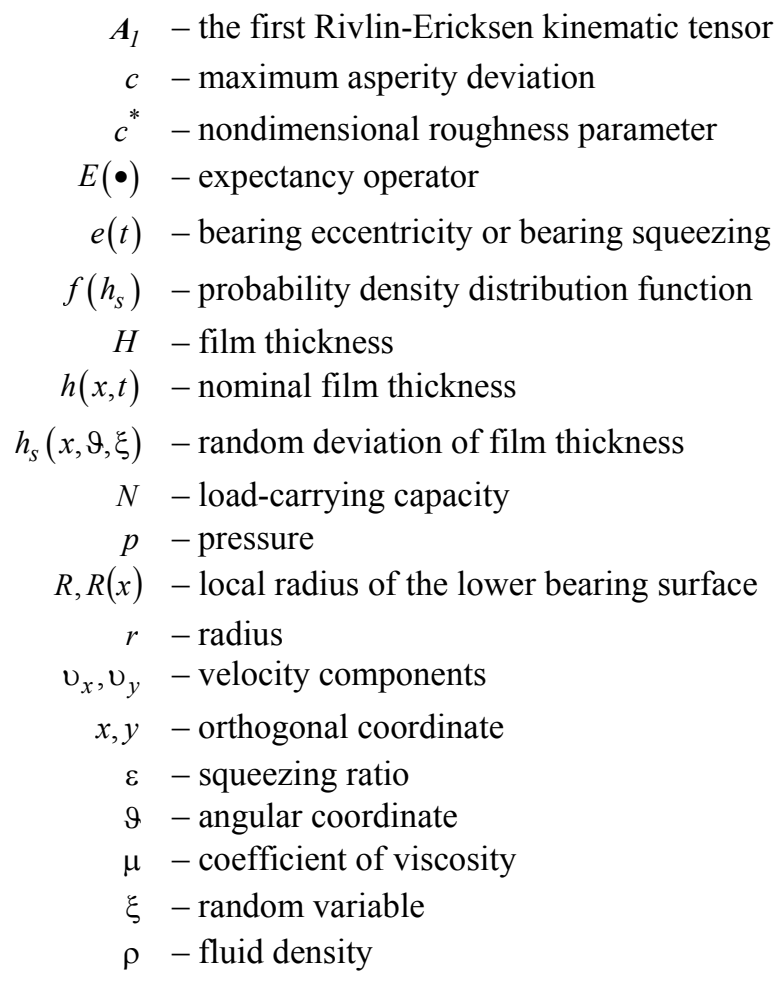

\section{Appendix}

For the probability density function given by Eq.(2.14) we have - for integer exponents - the following expected values of film thickness functions

$$
\begin{aligned}
& E(H)=h, \quad E\left(H^{2}\right)=h^{2}\left(1+\frac{1}{9} Y^{2}\right), \quad E\left(H^{3}\right)=h^{3}\left(1+\frac{1}{3} Y^{2}\right), \\
& E\left(H^{4}\right)=h^{4}\left(1+\frac{2}{3} Y^{2}+\frac{1}{33} Y^{4}\right), \quad E\left(H^{5}\right)=h^{5}\left(1+\frac{10}{9} Y^{2}+\frac{5}{33} Y^{4}\right),
\end{aligned}
$$




$$
\begin{aligned}
& E\left(H^{6}\right)=h^{6}\left(1+\frac{5}{93} Y^{2}+\frac{5}{11} Y^{4}+\frac{5}{429} Y^{6}\right), \\
& E\left(H^{-1}\right)=\frac{1}{h}\left\{\frac{35}{32} \frac{1}{Y^{7}}\left[\left(Y^{2}-1\right) \ln \frac{1+Y}{1-Y}-\frac{2}{15} Y\left(15-40 Y^{2}+33 Y^{4}\right)\right]\right\}, \\
& E\left(H^{-2}\right)=\frac{1}{h^{2}}\left\{\frac{35}{32} \frac{1}{Y^{7}}\left[6\left(Y^{2}-1\right) \ln \frac{1+Y}{1-Y}-\frac{4}{5} Y\left(15-25 Y^{2}+8 Y^{4}\right)\right]\right\}, \\
& E\left(H^{-3}\right)=\frac{1}{h^{3}}\left\{\frac{35}{32} \frac{1}{Y^{7}}\left[3\left(5-Y^{2}\right)\left(Y^{2}-1\right) \ln \frac{1+Y}{1-Y}+2 Y\left(15-13 Y^{2}\right)\right]\right\}, \\
& E\left(H^{-4}\right)=\frac{1}{h^{4}}\left\{\frac{35}{32} \frac{1}{Y^{7}}\left[4\left(5-3 Y^{2}\right) \ln \frac{1+Y}{1-Y}-\frac{8}{3} Y\left(15-4 Y^{2}\right)\right]\right\}, \\
& E\left(H^{-5}\right)=\frac{1}{h^{5}}\left\{\frac{35}{32} \frac{1}{Y^{7}}\left[3\left(Y^{2}-5\right) \ln \frac{1+Y}{1-Y}+\frac{2 Y}{1-Y^{2}}\left(15-13 Y^{2}\right)\right]\right\}, \\
& E\left(H^{-6}\right)=\frac{1}{h^{6}}\left\{\frac{35}{32} \frac{1}{Y^{7}}\left[3 \ln \frac{1+Y}{1-Y}-\frac{2 Y}{5\left(1-Y^{2}\right)}\left(15-25 Y^{2}+8 Y^{4}\right)\right]\right\}
\end{aligned}
$$

where

$$
Y=\frac{c}{h}
$$

The expected values of film thickness functions $E\left(H^{-i}\right), i=1,2, \ldots, 6$, are not suitable for numerical purposes for small $Y$ values, therefore Taylor expansions in powers of $Y$ are proposed

$$
\begin{aligned}
& E\left(H^{-1}\right)=\frac{1}{h}\left\{1+\sum_{n=1}^{\infty} \frac{105 Y^{2 n}}{(2 n+1)(2 n+3)(2 n+5)(2 n+7)}\right\}, \\
& E\left(H^{-2}\right)=\frac{1}{h^{2}}\left\{1+\sum_{n=1}^{\infty} \frac{105 Y^{2 n}}{(2 n+3)(2 n+5)(2 n+7)}\right\}, \\
& E\left(H^{-3}\right)=\frac{1}{h^{3}}\left\{1+\sum_{n=1}^{\infty} \frac{105(n+1) Y^{2 n}}{(2 n+3)(2 n+5)(2 n+7)}\right\}, \\
& E\left(H^{-4}\right)=\frac{1}{h^{4}}\left\{1+\sum_{n=1}^{\infty} \frac{35(n+1) Y^{2 n}}{(2 n+5)(2 n+7)}\right\},
\end{aligned}
$$




$$
\begin{aligned}
& E\left(H^{-5}\right)=\frac{1}{h^{5}}\left\{1+\sum_{n=1}^{\infty} \frac{35(n+1)(n+2) Y^{2 n}}{2(2 n+5)(2 n+7)}\right\}, \\
& E\left(H^{-6}\right)=\frac{1}{h^{6}}\left\{1+\sum_{n=1}^{\infty} \frac{7(n+1)(n+2) Y^{2 n}}{2(2 n+7)}\right\} .
\end{aligned}
$$

The first three formulae $(i=1,2,3)$ were given by Gururajan and Prakash (1999), the others - by Walicka (2012).

For fractional exponents we have

$$
\begin{aligned}
& E\left(H^{m}\right)=h^{m}\left\{1+\sum_{n=1}^{\infty} \frac{105 Y^{2 n}}{2 n !(2 n+1)(2 n+3)(2 n+5)(2 n+7)} \prod_{i=1}^{2 n}(m-i+1)\right\}, \\
& E\left(H^{-m}\right)=\frac{1}{h^{m}}\left\{1+\sum_{n=1}^{\infty} \frac{105 Y^{2 n}}{2 n !(2 n+1)(2 n+3)(2 n+5)(2 n+7)} \prod_{i=1}^{2 n}(m+i-1)\right\} .
\end{aligned}
$$

\section{References}

Bujurke N.M., Kudenatti R.B. and Awati V.B. (2007): Effect of surface roughness on squeeze film poroelastic bearings with special reference to synovial joints. - Mathematical Biosciences, vol.209, pp.76-89.

Christensen H. (1970): Stochastic model for hydrodynamic lubrication of rough surfaces. - Proc. Inst. Mech. Engrs, vol.184, pt 1, pp.1013-1022.

Christensen H. and Tønder K. (1971): The hydrodynamic lubrication of rough bearing surfaces of finite width. ASME, J. Lubric. Technol., vol.93, No.2, pp.324-330.

Christensen H. and Tønder K. (1973): The hydrodynamic lubrication of rough journal bearings. - ASME, J. Lubric. Technol., vol.95, No.1, pp.166-172.

Covey G.H. and Stanmore B.R. (1981): Use of the parallel-plate plastometer for the characterisation of viscous fluids with a yield stress. - J. Non-Newton. Fluid Mech. vol.8, pp.249-260.

Dai G. and Bird R.B. (1981): Radial flow of Bingham fluid between two fixed circular disks. - J. Non-Newton. Fluid Mech. vol.8, pp.349-355.

Dorier C. and Tichy J. (1992): Behaviour of a Bingham-like viscous fluid in lubrication flows. - J. Non-Newt. Fluid Mech., vol.45, No.3, pp.291-350.

Ellis S.B. (1927): Thesis, Lafayette College, Pa. Citted in: Matsuhisa S., Bird R.B. (1965): Analytical and numerical solutions for laminar flow of the Non-Newtonian Elis fluid. - AiChE Journal, 588-595.

Gururajan K. and Prakash J. (1999): Surface roughness effects in infinitely long porous journal bearing. - Journal of Tribology, Trans. ASME, vol.121, No.1, pp.139-147.

Khonsari M.M. and Dai F. (1992): On the mixture flow problem in lubrication of hydrodynamic bearing: small solid volume fraction. - STLE Trib. Trans., vol.35, No.1, pp.45-52.

Lin J.-R. (2000): Surfaces roughness effect on the dynamic stiffness and damping characteristics of compensated hydrostatic thrust bearings. - Int. J. Machine Tools Manufact., vol.40, pp.1671-1689,.

Lin J.-R. (2001): The effect of couple stresses in the squeeze film behavior between isotropic rough rectangular plates. - Int. J. Appl. Mech. Eng., vol.6, No.4, pp.1007-1024. 
Lipscomb C.C. and Denn M.M. (1984): Flow of Bingham fluids in complex geometries. - J. Non-Newt. Fluid Mech., vol.14, No.3, pp.337-349.

Morgan V.T. and Cameron A. (1957): Mechanismus of lubrication in porous metal bearings. - Proc. Conf. on Lubrication and Wear, Inst. Mech. Eng., London 1957, pp.151-157.

Prakash J. and Tiwari K. (1984): An analysis of the squeeze film between rough porous rectangular plates with arbitrary porous wall thickness. - Journal of Tribology, Trans. ASME, vol.106, No.2, pp.218-222.

Prakash J. and Tiwari K. (1985): Effects of surface roughness on the squeeze film between rectangular porous annular disc with arbitrary porous wall thickness. - Int. J. Mech. Sci., vol.27, No.3, pp.135-144.

Rajalingam C., Rao B.V.A. and Prabu S. (1978): The effect of a non-Newtonian lubricant on piston ring lubrication. Wear, vol.50, pp.47-57.

Rotem Z. and Shinnar R. (1961): Non-Newtonian flow between parallel boundaries in linear movements. - Chem. Eng. Sie., vol.15, pp.130-143.

Swamy S.T.N., Prabhu B.S. and Rao B.V.A. (1975): Stiffness and damping characteristics of finite width journal bearing with a non-Newtonian film and their application to instability prediction. - Wear, vol.32, pp.379-390.

Wada S. and Hayashi H. (1971): Hydrodynamic lubrication of journal bearings by pseudo-plastic lubricants. Pt 1, Theoretical studies, Pt 2, Experimental studies. - Bull. JSME, vol.14, No.69, pp.268-286.

Walicka A. (1989): Accurate and Asymptotic Solution of Simplified Sets of Equations Describing the Motion of Viscous Fluids in a Slot Bounded by Two Co-axial Surfaces of Revolution (in Polish). - Warszawa: PWN.

Walicka A. (1994): Micropolar flow in a Slot Between Rotating Surfaces of Revolution. - Zielona Góra: TU Press.

Walicka A. (2002): Rotational Flows of Rheologically Complex Fluids in Thin Channels (in Russian). - Zielona Gora: University Press.

Walicka A. (2009): Surface roughness effects in a curvilinear squeeze film bearing lubricated by a power-law fluid, Int. J. Appl. Mech. Engng, vol.14, No.1, pp.277-293.

Walicka A. (2012): Porous curvilinear squeeze film bearing with rough surfaces lubricated by a power-law fluid. Journal of Porous Media, vol.15, No.1, pp.29-49.

Walicka A. and Walicki E. (2002a): Surface roughness effect on the pressure distribution in curvilinear thrust bearings. - Exploitation Problems of Machines, vol. 131, No.3, pp.157-167.

Walicka A. and Walicki E. (2002b): Couple stress and surface roughness effects in curvilinear thrust bearings, Int. J. Appl. Mech. Engng, vol.7, Spec. Issue: SITC, pp.109-117.

Walicki E. (1975): Viscous fluid flow in a slot of the curvilinear thrust bearing. - Rev. Roum. Sci. Techn. - Mech. Appl., vol.20, No.4, pp.483-493.

Walicki E. (1977): Viscous Fluid Flow in Slots of Thrust Bearings. - Bydgoszcz: AT-R. Press.

Walicki E. (2005): Rheodynamics of Slide Bearings Lubrication (in Polish). - Zielona Gora: University Press.

Walicki E. and Walicka A. (1998): Mathematical modelling of some biological bearings. - Smart Materials and Structures, Proc. 4th European and $2^{\text {nd }}$ MiMR Conference, Harrogate, UK, 6-8 July 1998, pp.519-525.

Whorlow R.W. (1992): Rheological Techniques (Sec. Edition), Ellis Horwood, New York.

Received: July 5, 2014

Revised: August 12, 2014 